\title{
Bacteriemia por Neisseria subflava en un recién nacido
}

\author{
M. Carolina Rivacoba, Giannina Izquierdo, Natalia Zenteno y Lorena Porte
}

\section{Neisseria subflava bacteremia in newborns: case report and review of the literature}

Neisseria subflava belongs to Neisseriaceae family, is considered a comensal specie, however in certain host, mainly inmunosuppresed patientes and children, the literature has documented invasive infections. We present a case of a bacteriemia due to $N$. subflava in a newborn, treated with cefotaxime with good outcome. In newborns, the most common Neisseria bacteria to cause invasive infections are $N$. meningitidis, with highly fatal clinical course and $N$. gonorrhoeae which compromise the eye, oftalmia neonatorum, with uncommon invasive infections. It's very important the adequate microbiological diagnosis because the biochemical tests may be inconclusive. MALDITOF mass spectrometry technique is a useful tool.

Key words: Neisseria subflava, newborn, bacteremia.

Palabras clave: Neisseria subflava, recién nacido, bacteriemia.

\section{Introducción}

$N$ Teisseriaceae es una familia compuesta por diplococcos gramnegativos que pueden colonizar el tracto respiratorio y genitourinario del ser humano. Existen especies que habitualmente se asocian a enfermedad como Neisseria meningitidis y Neisseria gonorrhoeae. Sin embargo, algunas de las especies consideradas comensales, como Neisseria flavescens, $N$. mucosa, $N$. sicca, $N$. lactamica y $N$. subflava, se han asociado a infecciones en hospederos sanos, inmunocomprometidos y en pacientes sometidos a peritoneodiálisis ${ }^{1,2}$. Neisseria subflava con sus biovariedades N. subflava, $N$. perflava y $N$. flava ${ }^{3}$, ha sido descrita como agente causal de bacteriemia, meningitis y endocarditis en niños, con una mortalidad sobre $30 \%$. En algunos casos, se asocia a exantema petequial hemorrágico y púrpura. Neisseria subflava se ha descrito en forma esporádica como agente causal de artritis séptica en niños ${ }^{4}$.

En recién nacidos (RN) se ha aislado excepcionalmente, encontrándose sólo dos casos publicados en la literatura científica de bacteriemia y meningitis ${ }^{5,6}$.

Presentamos un caso de bacteriemia por N. subflava en un RN.

\section{Caso clínico}

$\mathrm{RN}$ que nace de un embarazo controlado, hijo de madre con sífilis diagnosticada durante el primer trimestre del embarazo con VDRL positivo en título 1:16 y MHA-TP reactivo. Fue tratada con tres dosis de penicilina benzatina con descenso de los títulos de VDRL a 1:2; sin embargo, tuvo una reinfección con ascenso de títulos a 1:8, por lo que se volvió a indicar tratamiento, alcanzando a recibir sólo una dosis de penicilina benzatina previa al parto.

El RN nació por parto vaginal a las 38 semanas de gestación, con un peso de nacimiento de $3,8 \mathrm{~kg}$. El VDRL del RN fue positivo con un título de 1:2 por lo que se internó en la unidad de neonatología con diagnóstico de sífilis congénita probable, para estudio y tratamiento. Se realizó un hemograma con 17.000 leucocitos $/ \mathrm{mm}^{3}$, sin desviación a izquierda, una PCR de 4,3 mg/L y una punción lumbar (PL) cuyo citoquímico del LCR fue normal y el VDRL en LCR fue no reactivo. Se inició tratamiento con penicilina sódica i.v. $50.000 \mathrm{UI} / \mathrm{kg}$ fraccionado cada $12 \mathrm{~h}$, según norma ministerial ${ }^{7}$.

Aproximadamente, a las $36 \mathrm{~h}$ de vida presentó un episodio de fiebre $\left(38,1^{\circ} \mathrm{C}\right)$ por lo que se controlaron parámetros inflamatorios. Destacó un viraje de la PCR a $32 \mathrm{mg} / \mathrm{L}$, sin cambios significativos en el hemograma. Se tomaron dos hemocultivos automatizados (Becton Dickinson) por venopunción en sitios distintos, con un volumen de muestra de $1 \mathrm{ml}$ cada uno. El paciente se mantuvo con terapia con penicilina, en buenas condiciones generales y sin fiebre. Ambos hemocultivos resultaron positivos a las $8 \mathrm{~h}$ de incubación. En la tinción de Gram se observaron diplococos gramnegativos y en el cultivo hubo desarrollo de colonias oxidasa positivas (Figuras 1 y 2). Las pruebas bioquímicas convencionales no fueron concluyentes, por lo que se realizó una espectrometría de masas (MALDITOF MS Microflex LT, Bruker ${ }^{\circledR}$ ), que identificó a la cepa como Neisseria subflava biovar perflava con $98,9 \%$ de certeza. Como el cultivo del primer
Universidad de Chile, Santiago. Programa de Formación de Infectología Pediátrica (MCR).

Hospital de niños Dr. Exequiel González Cortés, Santiago. Unidad de Infectología (GI) Hospital Barros Luco Trudeau, Santiago. Laboratorio de Microbiología (NZ). Clínica Alemana, Santiago. Laboratorio Clínico (LP)

Recibido: 9 de mayo de 2017 Aceptado: 1 de agosto de 2017

Correspondencia a: M. Carolina Rivacoba R. carorivacoba@gmail.com 
Figura 1. Placa de agar chocolate que muestra desarrollo de colonias circulares, convexas, brillantes, de aspecto amarillo-café.
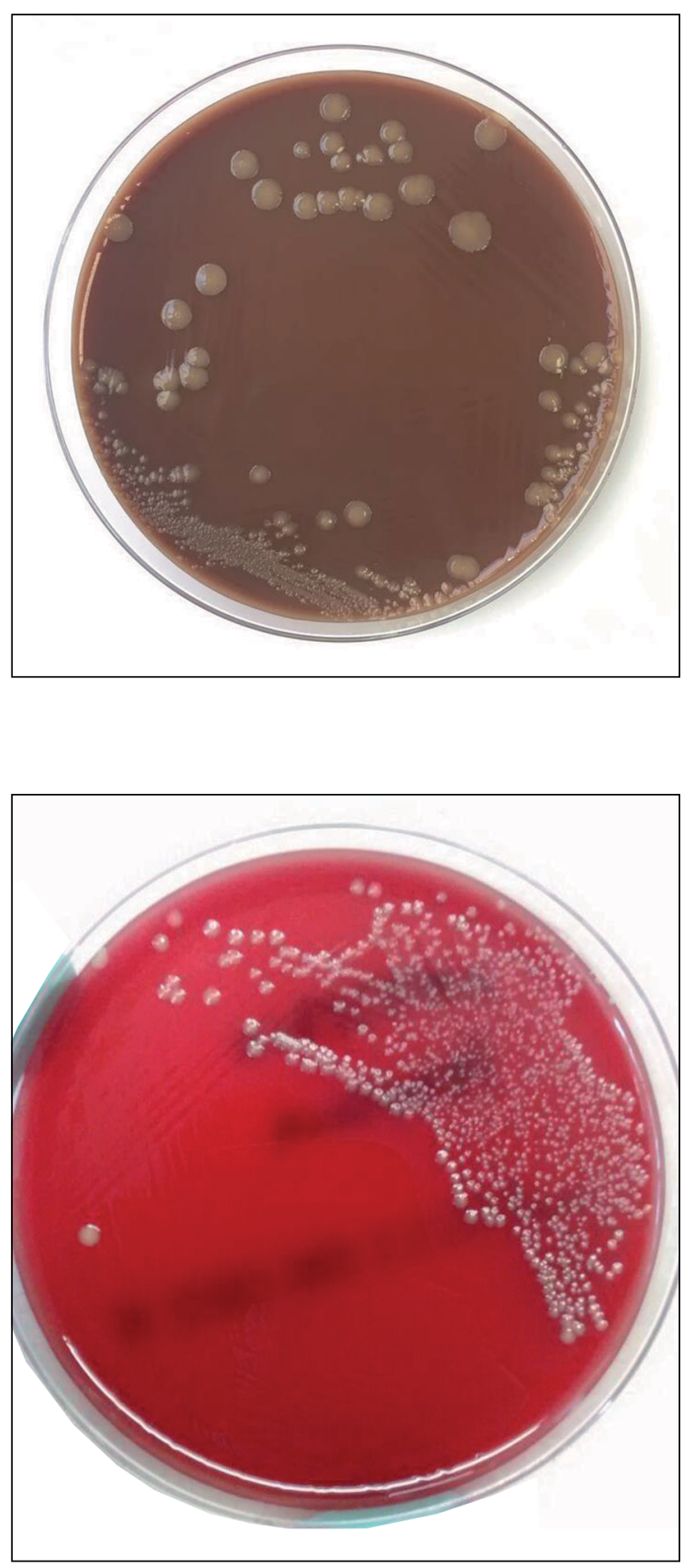

LCR fue negativo, se intentó realizar una segunda PL que resultó frustra. La identificación fue confirmada por el Laboratorio de Referencia Nacional (Instituto de Salud Pública). Respecto al estudio de susceptibilidad, se hizo estudio de $\beta$-lactamasas que fue negativa. No se realizó en el Laboratorio de Referencia estudio de susceptibilidad a otros antibacterianos. Recibió terapia con cefotaxima $150 \mathrm{mg} / \mathrm{kg} /$ día, fraccionada cada $8 \mathrm{~h}$ por 7 días. El paciente evolucionó siempre en buenas condiciones generales, afebril, sin desarrollar focos sépticos aparentes, dándose de alta a los 11 días de vida. En los controles ambulatorios por sífilis congénita probable se encontró asintomático y con VDRL no reactivo.

\section{Discusión}

La familia Neisseriaceae incluye tres géneros: Neisseria, Kingella y Eikenella. Las especies del género Neisseria se dividen en patógenas ( $N$. meningitidis y $N$. gonorrhoeae) y comensales. Éstas últimas fueron descritas en 1906 por von Lingelsheim, quién identificó $N$. cinerea, $N$. sicca, N. flava y N. subflava. Posteriormente, se identificó $N$. lactamica, que se relaciona estrechamente con N. meningitidis ${ }^{8}$.

Las bacterias del género Neisseria consideradas comensales aparecen precozmente en la cavidad oral y nasofaríngea. Estudios realizados en lactantes demuestran una colonización en las primeras semanas de vida, la que aumenta con la edad ${ }^{9}$. En ocasiones pueden actuar como patógenos oportunistas, especialmente en hospederos inmunocomprometidos o a consecuencia de mordeduras de animales ${ }^{10}$.

Neisseria subflava, presenta tres biovariedades (bv): $N$. subflava bv flava, bv. subflava y bv. perflava. Este agente se ha aislado desde la nasofaringe y el tracto genitourinario, considerándose una causa infrecuente de infección invasora, describiéndose casos de meningitis, endocarditis, conjuntivitis, artritis y bacteriemia ${ }^{1,2,11-13}$.

El aislamiento de este agente en hemocultivos debe interpretarse cautelosamente en base a los antecedentes clínicos, ya que es difícil discriminar entre contaminación e infección. Si se aisla en más de un hemocultivo sugiere una bacteriemia significativa ${ }^{14}$.

Existen pocos casos publicados en la literatura médica especializada, entre ellos nueve casos de menigitis por $N$. subflava, (de ellos, siete niños) sin descripción del biovar $^{12}$.

En otra serie publicada, se describen 16 casos pediátricos ocurridos entre los años 1963 y 2004, donde 13 eran niños sanos sin enfermedad subyacente y los restantes, correspondieron a niños con endocarditis y enfermedad cardiológica previa. Hubo seis fallecidos en este grupo, representando $38 \%$ de los pacientes. Cabe destacar que en estos casos se utilizó terapia antimicrobiana con sulfadiacina o penicilina ${ }^{1,11}$.

Los otros casos corresponden a un RN con un día de vida con bacteriemia y meningitis por $N$. subflava que evolucionó con un síndrome de Waterhouse Friderichsen, de curso fatal ${ }^{5}$, y otro RN con 7 días de vida que falleció por una meningitis en que se identificó $N$. flava ${ }^{6}$. En 
nuestro caso clínico, la única manifestación del RN fue fiebre, descartándose el compromiso meníngeo, con una evolución clínica favorable.

Desde el punto de vista microbiológico, N. subflava se visualiza en la tinción de Gram como cocáceas en pares y tétradas, con tendencia a resistir a la decoloración del Gram. Sus colonias producen un pigmento amarillento y no muestran hemólisis en el agar sangre; son lisas, transparentes $\mathrm{u}$ opacas, a menudo adherentes y frecuentemente aglutinan espontáneamente en solución salina. Utiliza glucosa y maltosa para producir ácido y no lactosa y manosa. Es oxidasa positiva y reduce los nitritos pero no los nitratos ${ }^{15}$. Se pueden diferenciar de otras especies por su capacidad de producir pigmento amarillo y de crecer a $22{ }^{\circ} \mathrm{C}^{13,16,17}$. Muchas de las especies saprofíticas exhiben resistencia enzimática a penicilinas mediada por $\beta$-lactamasas codificadas por TEM-1 gen tipo bla. Cuando ésta se produce en nivel bajo, se logra inhibir con acción de ácido clavulánico y sulbactam. Por otra parte, en dos cepas de $N$. perflava productoras de $\beta$-lactamasa se ha reportado existencia de plasmidos de 5,4 y 53,7 $\mathrm{Kb}$ que no se han documentado en $N$. meningitidis y $N$. gonorrhoeae $^{18}$. La resistencia a penicilina en Neisseria spp. comensales ascendería a 34\% y la documentación de penicilinasa de tipo bla TEM-1 llega a 9\%. Nuestro caso es concordante con la literatura médica, pues el neonato desarrolló la bacteriemia mientras se encontraba en tratamiento con penicilina.

El diagnóstico diferencial debe ser enfrentado con otras Neisserias spp. patógenas y comensales. Neisseria meningitidis se ha asociado a enfermedad invasora en RN con una incidencia de 9/100.000 RN, más baja que a otras edades, probablemente por el traspaso de anticuerpos maternos. Ocurre generalmente en madres que presentan colonización nasofaríngea o genitourinaria por N. meningitidis $^{19}$. Clínicamente se presenta en forma inespecífica, con fiebre y desarrollo de shock y falla orgánica múltiple a las pocas horas. Se describe una mortalidad de $40 \%$ en aquellos pacientes que presentan la infección durante la primera semana de vida ${ }^{19}$. Cobra importancia la identificación adecuada de la especie en el laboratorio, dado que N. subflava y N. meningitidis presentan un biotipo similar de fermentación de los azúcares ${ }^{11}$. Dado que estas especies pueden confundirse, es posible que $N$. subflava estuviese subdiagnosticada. Además, N. subflava no está disponible en todas las bases de datos de sistemas automatizados; siendo la espectrometría de masas una técnica de utilidad en su identificación ${ }^{20}$.

La infección por $N$. gonorrhoeae se presenta en este grupo etario y la oftalmia neonatorum es la principal forma de manifestación clínica. Se describe entre 2 y $48 \%$ de los RN que no reciben profilaxis ocular, pudiendo progresar a úlceras corneales, endoftalmitis, ceguera e incluso enfermedad invasora como sepsis, meningitis o artritis, entre otras ${ }^{21}$. Se han descrito infecciones invasoras por $N$. gonorrhoeae en menos de 1\% de los RN con infección perinatal; la artritis séptica es la forma diseminada más frecuente. La sepsis por $N$. gonorrhoeae es generalmente silente en su fase bacteriémica, ocurriendo más frecuentemente en RN prematuros. La meningitis por este agente es muy infrecuente, aunque es recomendable descartarla ante una infección localizada o sistémica ${ }^{18,19}$.

Como conclusión, N. subflava biovar perflava, considerado un microorganismo comensal, puede actuar como patógeno y causar enfermedad invasora en $\mathrm{RN}$ y pacientes inmunocomprometidos. Dentro de su perfil de susceptibilidad destaca más de $30 \%$ de resistencia a penicilina, por lo que como esquema antimicrobiano debe ser considerada una cefalosporina de tercera generación.

\section{Resumen}

Neisseria subflava es una especie considerada comensal dentro de la familia Neisseriaceae; sin embargo, en algunos hospederos inmunocomprometidos $\mathrm{y}$ en niños se ha documentado como agente causal de infecciones invasoras. Se presenta el caso clínico de un recién nacido con bacteriemia por $N$. subflava con buena respuesta a terapia con cefotaxima. Las bacterias del género Neisseria más frecuentes de causar infecciones en recién nacidos son $N$. meningitidis, de alta mortalidad y $N$. gonorrhoeae, que se manifiesta principalmente con oftalmia neonatorum. Se destaca la importancia de realizar un diagnóstico microbiológico adecuado dado que las pruebas biquímicas pueden ser no concluyentes. La técnica de espectrometría de masas MALDITOF resulta una herramienta de utilidad.

\section{Referencias bibliográficas}

1.- Demmler G J, Couch R S, Taber L H. Neisseria subflava bacteremia and meningitis in a childreport of a case and review of the literature. Pediatr Infect Dis 1985; 4: 286-8.

2.- Pollack S, Mogtader A, Lange M. Neisseria subflava endocarditis. Case report and review of the literature. Am J Med 1984; 76: 752-8.
3.- Johannes E F M. Neisseria. Manual of Clinical Microbiology. Jorgensen JH, Pfaller MA et al, eds. ASM Press, Washington D, editor. 11th ed. 2015. p 635-51.

4.- Obeid E M. Neisseria subflava causing arthritis of the ankle in a child. J Infect 1993; 27: 100-1.

5.- Martínez-Tallo E, Hernández-Rastrollo R, Benito-Alonso E, Cordero-Carrasco J L Cardesa-García J J. Meningitis neonatal por
Neisseria subflava. An Esp Pediatr 1988; 29: 475-6.

6.- Noguchi T T, Nachum R LC. Acute purulent meningitis caused by chromogenic Neisseria: A case report and literature review. Med Arts Sci 1963; 11: 11-8.

7.- Norma General Técnica No 0141 de 2012, Ministerio de Salud. Norma Conjunta de Prevención de la Transmisión Vertical del 
VIH y la Sífilis 2012. http://www.minsal. cl/portal/url/item/d84c1b1497766e4 8e040010164010137.pdf (Fecha de acceso: 20 de enero de 2017).

8.- Liu G, Tang C M, Exley R M. Non-pathogenic Neisseria: members of an abundant, multihabitat, diverse genus. Microbiology 2015; 161: 1297-312.

9.- McCarthy C, Snyder M L, Parker R B. Oral flora of man-i. The newborn to the 1-year-old infant. Arch Oral Biol 1965; 10: 61-70.

10.- Johnson A P. The pathogenic potential of commensal species of Neisseria. J Clin Pathol 1983; 36: 213-23.

11.- Tena Gómez D, Carranza González R, Torres Cañadillas C, Manrique González E, Garrido Palomo R. Bacteriemia por Neisseria subflava biovar flava. An Pediatr (Barc) 2004; 61: 188-9.

12.- Baraldès M A, Domingo P, Barrio J Ll, Pericas R, Gurguí M, Vazquez G. Meningitis due to Neisseria subflava: case report and review. Clin Infect Dis 2011; 30: 615-7.

13.- Chong Y, Song K S. Lee S Y. Neisseria subflava infections: bacteriological aspects of two cases. Yonsei Med J 1975; 16: 44-9.

14.- Feder H M, Garibaldi R A. The significance of nongonococcal, nonmeningococcal Neisseria isolates from blood cultures. Rev Infect Dis 1984; 6:181-8.

15.- Services M. UK Standards for microbiology investigations. Bacteriology 2015; B 55 (5.2): 1-21.

16.- Tan A, Hill D M, Harrison O B, Srikhanta Y N, Jennings M P, Maiden M C, et al. Distribution of the type III DNA methyltransferases modA, modB and modD among Neisseria meningitidis genotypes: implications for gene regulation and virulence. Sci Rep 2016; 6: 21015.

17.- Mechergui A, Achour W, Ben Hassen A. Laboratory identification methods of nonpathogenic Neisseria species. Rev Med Microbiol 2014; 25: 85-92.
18.- Mechergui A, Achour W, Ben Hassen A. Antibiotic resistance among commensal Neisseria species. Rev Med Microbiol 2014; 25: 93-9.

19.- Kiray Baş E, Bülbül A, Cömert S, Uslu S, Arslan S, Nuhoglu A. Neonatal infection with Neisseria meningitidis: analysis of a 97-year period plus case study. J Clin Microbiol 2014; 52: 3478-82.

20.- Jamal W, Albert M J, Rotimi V O. Realtime comparative evaluation of bioMerieux VITEK MS versus Bruker Microflex MS, two matrix-assisted laser desorption-ionization time-of-flight mass spectrometry systems, for identification of clinically significant bacteria. BMC Microbiol 2014; 14: 289.

21.- Woods C R. Gonococcal infections in neonates and young children. Semin Pediatr Infect Dis 2005; 16: 258-70.

22.- Moore D L, MacDonald N E. Preventing ophthalmia neonatorum. Can J Infect Dis Med Microbiol 2015; 26: 122-5. 